JGG 2020;68:224-230

doi: 10.36150/2499-6564-271

\title{
Treatment of COVID-19 disease in older people with cognitive impairment: a challenge into the challenge
}

Anna Maria Martone, Francesca Ciciarello, Francesco Landi, for the Gemelli Against COVID-19 Geriatric Team*

* Gemelli Against COVID-19 Geriatric Team: F. Landi, C. Barillaro, A. Bellieni, V. Brandi, A. Carfi, M.C. Cipriani, E D’Angelo, C. Falsiroli, D. Fusco, G. Landi, R. Liperoti, M.R. Lo Monaco, A.M. Martone, E. Marzetti, F. Pagano, C. Pais, A. Russo, S. Salini, M. Tosato, A.M. Tummolo, N. Acampora, F. Benvenuto, G. Bramato, L. Catalano, F. Ciciarello, I. Martis, S. Rocchi, E. Rota, A. Salerno, M. Tritto, A. Sgadari, G. Zuccalà, and R. Bernabei

Received: September 28, 2020

Accepted: December 9, 2020

\section{Correspondence}

Francesco Landi

Fondazione Policlinico Universitario "Agostino Gemelli" IRCCS, Università Cattolica del Sacro Cuore, L.go F. Vito 1, 00168 Rome, Italy

E-mail: francesco.landi@unicatt.it

\section{Conflict of interest}

The Authors declare no conflict of interest

How to cite this article: Martone AM, Ciciarello $F$, Landi $F$, et al. Treatment of COVID-19 disease in older people with cognitive impairment: a challenge into the challenge. Journal of Gerontology and Geriatrics 2020;68:224-30. https://doi. org/10.36150/2499-6564-271

(C) Copyright by Società Italiana di Gerontologia e Geriatria (SIGG)

\section{(c) (1) $(9)$}

\section{OPEN ACCESS}

This is an open access article distributed in accordance with the CC-BY-NC-ND (Creative Commons Attribution-NonCommercial-NoDerivatives 4.0 International) license. The article can be used by giving appropriate credit and mentioning the license, but only for non-commercial purposes and only in the original version. For further information: https://creativecommons.org/licenses/by-nc-nd/4.0/deed.en
The World Health Organization (WHO) has declared COVID-19 disease a public health emergency of international concern. Even though no age-group is safe from the SARS-CoV-2 infection, the burden is extremely higher and most severe among persons aged 70 years and over, with a well-known mortality rates of more than $20 \%$ among octogenarians. The presence of multiple pre-existing comorbidities is associated with more severe SARS-CoV-2 infection manifestation, reflecting the presence of pre-existent physical and/or cognitive frailty.

One of the main problems in patients with cognitive disorders is the management of drug therapy. In fact, there are a number of interactions and contraindications in the simultaneous administration of the antiviral drugs and the drugs that are commonly taken by older people. The aim of this review is to provide a clear and simple indication on how to improve the pharmacological and non-pharmacological management of patients with dementia and at the same time suffering from COVID-19 disease.

Key words: COVID-19, dementia, drug therapy, drug interaction, personalized medicine

The coronavirus disease COVID-19 (SARS-CoV-2) outbreak has raised numerous challenges for health care systems and one of the most important of them, often forgotten, is to manage patients' psychiatric disorders safely, in addition to prevent and treat SARS-CoV-2 infection ${ }^{1}$. In fact, managing patients with severe psychiatric disorders, such as dementia, who are suspected or confirmed to be affected by COVID-19 disease, is a major logistical and clinical challenge ${ }^{2}$. In addressing this challenge, health systems need to re-balance their resources from hospital-centric services to community-based (such as, nursing home and rehabilitation services) and primary care services. The lesson and experience derived from this bio-disaster is extremely useful to better meet the needs of persons with cognitive impairment and dementia ${ }^{3}$.

\section{COVID-19 DISEASE IN PEOPLE WITH DEMENTIA}

Many older patients admitted to acute care hospital suffer from cognitive impairment (dementia or delirium) along with other comorbidities. Older 
patients, and mainly those with cognitive impairment, are at enhanced risk of delirium while waiting in emergency room and, afterward, during the hospital stay. Scientific evidence clearly demonstrates that delirium is correlated with serious short- and long-term negative outcomes, such as increased mortality, falls, functional and further cognitive decline ${ }^{4}$. Considering the need to adopt contact isolation when the risk of SARS-CoV-2 infection is posed (usually already on arrival in the ambulance to the emergency room), the frail older people with cognitive impairment are more exposed for sure to a psychological stress that cannot be correctly addressed. If we consider that this situation is the cause of important psychological disorders, such as anxiety, depression, panic attacks, even in younger people without a history of psychiatric disorders, it is possible to hypothesize how the management of the patient at risk and/or affected by COVID-19 disease is particularly complex in the demented patient.

It is well established that hospitalization per se and/or comorbidities can negatively affect cognitive function, but at the same time cognitive dysfunction affects hospital outcomes, too. In particular, patients who develop delirium during the hospital stay have both short-term and long-term poor outcomes (such as medications side effects, bed rest, pressure ulcers, incontinence, falls, increased length of stay, behavior problems, higher mortality), regardless of the underlying diseases or other important confounders.

For patients with cognitive impairment and delirium, it is important not to underestimate these problems and to identify and to treat the underlying causes ${ }^{5}$. Prompt screening, assessment and systematic diagnoses are crucial. As well as treating the underlying medical causes, the involvement of family members and a calm, safe and comfortable environment are important, too. In the situation of isolation that is created around the patient with suspicion of SARS-CoV-2 infection, the role of the geriatrician is of great importance in order to better manage these distress situations ${ }^{1}$. The infectious disease specialists and pulmonologists, who are the first to start treating patients with COVID-19 disease, are usually not "familiar" with the management of patients suffering from dementia and their all related problems. For this reason, the geriatrician should always be present in the medical staff who treat these patients ${ }^{1}$. Older patients with cognitive impairment should have person-centred care with the specific aim to early identify and to eventually minimize the specific risks of injury. The treatment should be always aligned with the patient's and family's preferences and appropriate for these specific circumstances.

\section{COVID-19 DISEASE TREATMENT APPROACHES}

Many studies have documented the potential effects of the antiviral drugs (darunavir, lopinavir, ritonavir and remdesivir) to reduce viral load and eventually to prevent the respiratory complications ${ }^{6-8}$ ). The therapeutic approach used tentatively to treat patients affected by COVID-19, as suggested by Italian Society of Infectious and Tropical Diseases guidelines (http://www.simit.org/ medias/1569-covid19), depends on the patient's clinical condition, age and presence or absence of comorbidity ${ }^{9,10}$. The latest update of the guidelines is May 5 , 2020.

A COVID-19 positive patient asymptomatic or with mild symptoms (fever $<37.5^{\circ} \mathrm{C}$, cough, symptoms similar to flu infection without dyspnea), age $<70$ years and without risk factors (i.e., COPD, diabetes and heart disease) and chest $X$-ray negative for pneumonia follows a clinical observation with supportive therapy.

A COVID-19 positive patient with mild respiratory symptoms but aged $>70$ years plus the presence of risk factors (i.e., COPD, diabetes and heart disease) or a chest $\mathrm{X}$-ray showing pneumonia should start: lopinavir/ ritonavir 200/50 mg, 2 tablets $\times 2$ /day (alternatively darunavir 800 mg 1 tablet/day + ritonavir 100 mg 1 tablet/day or darunavir/cobicistat 800/150 mg 1 tablet/ day), + chloroquine $500 \mathrm{mg}, 1$ tablet $\times 2$ /day or hydroxychloroquine $200 \mathrm{mg}, 1$ tablet $\times 2$ /day. Based on clinical evolution, duration of therapy should be from 5 to 20 days.

In case of need for oxygen therapy or a rapid clinical, it is recommended to suspend lopinavir/ritonavir (or darunavir) and continue with remdesivir $150 \mathrm{mg}$, first day 200 mg iv in 30 minutes then 100 mg iv per day for another 9 days, in combination with chloroquine 500 mg, 1 tablet $\times 2$ /day or hydroxychloroquine $200 \mathrm{mg}, 1$ tablet $\times 2$ /day. Based on clinical evolution, duration of therapy should be from 5 to 20 days.

Tocilizumab is a humanized antiinterleukin-6-receptor monoclonal antibody that inhibits interleukin- 6 signaling used as treatment of rheumatoid arthritis. Tocilizumab is administered intravenous experimentally in the treatment of COVID-19 disease in China and now in Italy with some encouraging results, especially in preventing the worsening of acute respiratory distress syndrome ${ }^{11}$. Finally, investigators of the COVID-19 RECOVERY trial documented in a press release that subjects with severe COVID-19 who have received $6 \mathrm{mg}$ dexamethasone once daily had an $8-26 \%$ lower mortality than participants who had received standard care ${ }^{12}$. However, it is important to highlight that, at the time of writing the present paper, the results remain neither peer reviewed nor fully published. 
Table I. Potential interaction between most frequently drug used for COVID-19 disease and CNS drugs (from Liverpool Drug Interactions website [https://www.covid19-druginteractions.org/prescribing-resources], mod.).

\begin{tabular}{|c|c|c|c|}
\hline & Darunavir/ritonavir & Lopinavir/ritonavir & Hydroxychloroquine \\
\hline \multicolumn{4}{|c|}{ ANTIDEPRESSANTS } \\
\hline Citalopram & $\boldsymbol{\oplus \Theta}$ & $\boldsymbol{\theta}$ & $\boldsymbol{\theta}$ \\
\hline Duloxetine & $\boldsymbol{\oplus \Theta}$ & $\boldsymbol{\oplus \Theta}$ & $\boldsymbol{\oplus \Theta}$ \\
\hline Escitalopram & $\boldsymbol{\oplus \Theta}$ & $\boldsymbol{\theta}$ & $\boldsymbol{\theta}$ \\
\hline Fluoxetine & $\boldsymbol{\oplus \Theta}$ & $\boldsymbol{\oplus \Theta}$ & $\oplus \oplus \oplus$ \\
\hline Mirtazapine & $\boldsymbol{\theta}$ & $\boldsymbol{\theta}$ & Ф๑๑ \\
\hline Trazodone & $\boldsymbol{\theta}$ & $\boldsymbol{\theta}$ & $\boldsymbol{\theta}$ \\
\hline Venlaflaxina & $\boldsymbol{\theta}$ & $\boldsymbol{\theta}$ & $\oplus \oplus \oplus$ \\
\hline \multicolumn{4}{|c|}{ ANTIPSYCHOTIC/NEUROLEPTICS } \\
\hline Clozapine & $\boldsymbol{\theta}$ & $\boldsymbol{\theta}$ & $\boldsymbol{\theta}$ \\
\hline Haloperidol & $\oplus \boldsymbol{\theta}$ & $\boldsymbol{\theta}$ & $\boldsymbol{\theta}$ \\
\hline Olanzapine & $\oplus \oplus \bullet$ & $\boldsymbol{\theta}$ & $\oplus \oplus \bullet$ \\
\hline Quetiapine & $\boldsymbol{\theta \theta \theta}$ & $\boldsymbol{\theta \theta \theta}$ & $\boldsymbol{\theta}$ \\
\hline Risperidone & $\boldsymbol{\theta}$ & $\boldsymbol{\theta}$ & $\boldsymbol{\theta}$ \\
\hline \multicolumn{4}{|c|}{ ANXIOLYTICS/HYPNOTICS/SEDATIVES } \\
\hline Alprazolam & $\boldsymbol{\theta}$ & $\boldsymbol{\theta}$ & $\oplus \oplus \oplus$ \\
\hline Bromazepam & $\oplus \theta$ & $\oplus \theta$ & Ф๑๑ \\
\hline Diazepam & $\boldsymbol{\theta}$ & $\boldsymbol{\theta}$ & Ф๑๑ \\
\hline Lorazepam & $\oplus \oplus \bullet$ & ๑๑७ & $\oplus \oplus \oplus$ \\
\hline Triazolam & $\boldsymbol{\theta \theta \theta}$ & $\boldsymbol{\theta \theta \theta}$ & Ф๑๑ \\
\hline Midazolam (oral) & $\boldsymbol{\theta \theta \theta}$ & $\boldsymbol{\theta \theta \theta}$ & $\oplus \oplus \oplus$ \\
\hline Zolpidem & $\boldsymbol{\theta}$ & $\boldsymbol{\theta}$ & ๑๑७ \\
\hline Zopiclone & $\boldsymbol{\theta}$ & $\boldsymbol{\theta}$ & $\oplus \oplus \bullet$ \\
\hline
\end{tabular}

\begin{tabular}{|l|l|}
\hline 00 & These drugs should not be co-administered
\end{tabular}

- Potential interaction - may require close monitoring, modification of drug dosage and/or timing of administration

$\oplus \odot$ Potential interaction - likely to be of weak intensity; additional action/monitoring or dosage adjustment is unlikely to be required

$\oplus \odot \odot$ No clinically significant interaction expected

\section{HOW DEMENTIA AFFECT COVID-19 TREATMENT DECISIONS: DRUG INTERACTIONS}

The use of drugs that are indicated as potential treatment of COVID-19 patients must be carefully evaluated in the patient affected by cognitive impairment, in particular for the potential and serious interactions with drugs that are likely to be already prescribed, such as drugs acting on the nervous system central (CNS). In this respect it is important to highlight that the Liverpool Drug Interaction Group, established in 1999 by members of the Department of Pharmacology at the University of Liverpool for the HIV disease, provides a freely available drug-drug interaction resource for drugs potentially used to manage the SARS-CoV-2 infection. The Liverpool Drug Interactions website (https://www. covid19-druginteractions.org/prescribing-resources) should be always checked to obtain updated information about the likelihood of interactions between the experimental agents used for the treatment of COVID-19 and commonly prescribed co-medications for dementia. Here we summarize the most common combination between drugs usually used in COVID-19 patients and drugs probably used for dementia.

\section{Possible INTERACTION BETWEen ANTIVIRAL DRUGS AND CNS DRUGS}

Lopinavir/ritonavir, commonly used for HIV treatment in adults, is used (off-label) for the treatment of COVID 19 related pneumonia ${ }^{13}$. Lopinavir and ritonavir are both CYP3 inhibitors, an isoform of the cytochrome P450. Therefore, these medications should not be 
co-administered with other medicines that are highly dependent on CYP3A for their clearance and for which high plasma concentrations are associated with severe events or mortality ${ }^{14}$.

Before prescribing these drugs, it is important to consider all the potential interactions with drugs acting at the central nervous system level.

- Valproic acid: the co-administration of lopinavir/ritonavir and valproic acid could reduce valproic acid serum levels ${ }^{15}$.

- Trazodone: the co-administration of lopinavir/ritonavir and trazodone increases the concentration of the trazodone from two to four times ${ }^{16}$. Adverse events such as nausea, dizziness, hypotension and syncope could be observed. The combination must be used with caution and must be considered the lowest dose of trazodone.

- Quetiapine: due to the inhibition of the CYP3A by lopinavir/ritonavir, concentrations of quetiapine could increase ${ }^{17}$. Co-administering lopinavir/ritonavir and quetiapine is contraindicated because it can increase toxicity related to quetiapine.

- Midazolam: the co-administration of lopinavir/ritonavir and midazolam increases the concentration of the midazolam 13 times with oral formulation, 4 times with parenteral formulation. Lopinavir/ritonavir have not to be administered with oral formulation midazolam while it is important to have particular attention to the co-administration of lopinavir/ritonavir and midazolam parenterally ${ }^{18}$. If lopinavir/ritonavir is co-administered with midazolam parenterally, this must be carried out in a intensive care unit (ICU) that guarantees a close clinical monitoring and an appropriate medical treatment in the case of respiratory depression.

- Triazolam: the co-administration of lopinavir/ritonavir and triazolam increases the concentration of triazolam and this combination should be avoided, too ${ }^{19}$.

In addition, particular caution and strict electrocardiography monitoring are also suggested during co-administration of drugs that lengthen the QT range, such as SSRI or SNRI antidepressants, antipsychotics and/or acetylcolinesterase inhibitors ${ }^{20}$.

Darunavir/ritonavir are CYP3A, CYP2D6 and P-gp inhibitors ${ }^{21}$. Co-administering darunavir/ritonavir with other medicines metabolized mainly by CYP3A and/or CYP2D6 or carried by P-gp may lead to an increase in systemic exposure of such medications that may increase or prolong their therapeutic effect and enhance the risk of side effects ${ }^{22}$.

In particular, when patients with cognitive disorders affected by COVID-19 disease are treated with these antiviral drugs, it is important to evaluate the risk of potential interactions with drugs acting at central nervous system level.

- Antidepressants: concurrent use of antidepressants (such as, paroxetine, sertraline, amitriptyline, desipramine, imipramine, nortriptyline, trazodone) can increase their plasma concentrations through CYP2D6 and/or CYP3A inhibition; therefore, a lower dose of these antidepressants should be considered ${ }^{19}$.

- Quetiapine: concurrent administration of darunavir/ low doses of ritonavir and quetiapine is contraindicated as it can increase the liver toxicity related to quetiapine metabolism through CYP3A inhibition.

- Sedatives/hypnotics: These drugs are widely
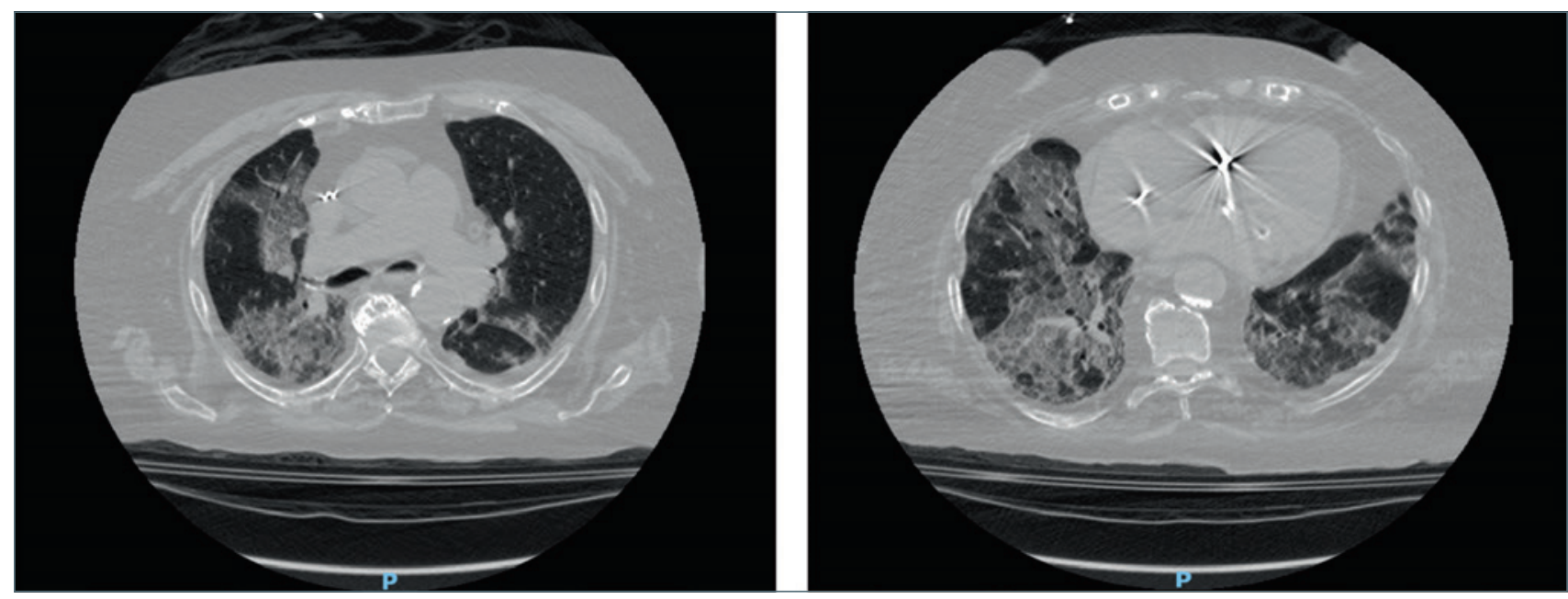

Figure 1. Chest CT scan shows diffuse ground-glass opacities, consolidation area, and both ground-glass opacities with consolidation in female, 87-year-old patient, living in nursing homes, suffering from dementia, type 2 diabetes mellitus, chronic kidney failure, atrial fibrillation, COPD, and heart failure. She was admitted to the Policlinico Gemelli Hospital for fever and dyspnea. Upon arrival in emergency room nasopharyngeal swab was performed showing positive result for SARS-CoV-2 infection. 
metabolized by CYP3A; therefore, co-administration with darunavir/ritonavir can cause a significant increase of their concentrations. In particular, the use of darunavir/ritonavir is contraindicated while patient is taking triazolam or oral midazolam.

\section{POSSIBLE INTERACTION BETWEEN HYDROXYCHLOROQUINE/ CHLOROQUINE AND CNS DRUGS}

The therapeutic action of hydroxychloroquine/chloroquine is based on several potential pharmacological effects, such as interaction with sulfidrilic groups, modulation of enzyme activity (in particular phospholirase, $\mathrm{NADH}$-cytochrome $\mathrm{C}$ reduttase, cholinesterase, protease), DNA fixation, stabilization of lysosomal membranes, inhibition of prostaglandin synthesis, polymorphonucleate chemotaxis and phagocytosis, possible interference with the production of interleukin 1 by monocytes and inhibition of the release of superoxide by neutrophils ${ }^{23,24}$. However, it is important to consider that hydroxychloroquine/chloroquine could prolong QT range. Chloroquine is known to be an open channel blocker of the hERG $1 \mathrm{~A}$ and $1 \mathrm{~A} / 1 \mathrm{~B}$ potassium channel that in the heart triggers the repolarizing potassium current $I_{K r}$. Therefore, particular caution and strict electrocardiography monitoring are suggested when such drug is co-administered with drugs that potentially lengthen the QT range, such as SSRI or SNRI antidepressants, antipsychotics or acetylcolinesterase inhibitors. More recently, Kassi and colleagues (25) clearly addressed that the increased oxidative injury caused by chloroquine could be responsible for the drug's toxicity. Taking into account that chloroquine-induced haemolysis is a result of reduced G6PD enzyme activity and that G6PD-deficient subjects may be more susceptible to human coronavirus, physicians may need to be more careful when treating COVID-19 patients with chloroquine, especially at large doses.

\section{Possible interaction betWeen tocilizumab and CNS DRUGS}

Tocilizumab specifically binds to both soluble and membrane IL-6 receptors and it has been shown to inhibit signals mediated by IL-6 receptors ${ }^{11}$. As known, IL-6 is a pro-inflammatory pleiotropic cytokine produced by different cell types, including $T$ and B cells, monocytes and fibroblasts. IL-6 is involved in several physiological processes, such as $T$ cell activation, immunoglobulin secretion induction, induction of acute phase protein liver synthesis and hemopoiesis stimulation. The expression of CYP450 liver enzymes is suppressed by cytokines, such as IL-6, which stimulate chronic inflammation. Therefore, the expression of CYP450 can be reversed when introducing a powerful cytokine inhibition therapy, such as tocilizumab. When starting or stopping tocilizumab therapy, patients being treated with medicines which are metabolized by CYP450 3A4, 1A2 or $2 \mathrm{C9}$, such benzodiazepines, should be monitored, as an increase in dose may be required to maintain the therapeutic effect. Finally, it is important to highlight that, considering its long elimination half-life, the effect of tocilizumab on the activity of the CYP450 enzyme may persist for several weeks after stopping therapy ${ }^{26}$.

\section{COMPLIANCE TO NON-PHARMACOLOGICAL SUPPORTIVE TREATMENT}

In addition to drug therapy, oxygen therapy represents a cornerstone in the treatment of the interstitial pneumonia COVID-19 related. In older patient with mild to severe cognitive impairment with associated behavioral disorders, compliance is often lacking. These patients often develop delirium during hospitalization and preventive isolation in which they are placed. The inability to see familiar faces, the subversion of the sleep-wake rhythm produces episodes of motor agitation that often leads the patient to remove oxygen masks and venous accesses. Knowing the drug interactions and the central effects of sedative drugs on the regulation of breathing, the use of these drugs is often not recommended. Nutrition is also a crucial node in the outcomes of these patients. Caloric and protein malnutrition, the inability to eat, the poor compliance with nutritional support devices such as a nasogastric tube, irreparably compromise the outcomes of these patients ${ }^{27,28}$.

\section{THE EXPERIENCE OF GEMELLI HOSPITAL AGAINST COVID-19}

Italy has been one of the largest and most serious clusters of SARS-CoV-2 infection in Europe. Despite the aggressive restraint and the total lock-down started during the first week of March 2020, the COVID-19 disease continued to increase day by day, reaching more than 240,000 of infected patients with one of the highest mortality rates (14\%).

When the number of infected people started to increase in the centre of Italy, the Fondazione Policlinico Gemelli IRCCS Teaching Hospital in Rome established a specific "track" in emergency room to early identify the patients at risk and/or confirmed to be affected by SARS-CoV-2 infection. In addition to the infectious diseases' units, the hospital organized seven other units for the observation and treatment of patients suspected and confirmed to be affected by COVID-19 disease. The first month experience (since the day which two elective surgical units were transformed into COVID-19 
units -50 beds - and were entrusted to a team of geriatricians), can be useful for all the hospitals in the world who are dealing with this emergency.

During this period, 110 patients were admitted to the COVID-19 geriatric units directly from the emergency room, including 75 (68\%) male and 35 (32\%) female. The mean age was $68.6+16.1$ years. The most frequent symptoms were fever (85\%), cough (50\%), dyspnoea (46\%), myalgia and/or arthralgia (15\%), fatigue (24\%), nausea (10\%), and diarrhea (5\%). 21 patients $(19 \%)$ of this simple were affected by dementia.

After performing two swabs (pharyngeal and nasal) repeated at a distance of 48 hours, 76 patients (57 male and 19 female) were found to be positive for COVID-19 at the first (72) or the second (4) pharyngeal or nasal swab. Among subjects positive for COVID-19, the mean number of diseases was $1.9+1.5$ and the most frequently observed diseases were hypertension (52\%), heart failure $(37 \%)$, renal failure $(30 \%)$, respiratory diseases $(26 \%)$, diabetes (12\%), and cancer (8\%). All patients suffered from interstitial pneumonia. Dementia of any type was present in $9(12 \%)$ patients. The onset of delirium during hospital stay was observed in $10 \%$ of patients.

The presence of a diagnosis of dementia and the onset of delirium are among the most serious problems that need to be addressed in the treatment of older patients with COVID-19 disease. First, the presence of psychomotor agitation and behavioral disorders makes difficult to perform diagnostic tests important for the management of these patients, such as arterial sampling for blood gas analysis, chest X-ray and CT scan. Chest $X$-ray may be useful as an initial screening, but even in patients with dementia, the chest CT scan (Fig. 1) is important to better evaluate the severity of the disease and better orientate the therapeutic choices. Furthermore, compliance with oxygen therapy is often very difficult to achieve as well as nutritional and hydration interventions. Overall, it is important to highlight that the management of these patients is further complicated by the difficulty of using drugs (such as antipsychotic and/or sedative medications) that potentially have interactions with the drugs indicated in the treatment of SARS-CoV-2 infection.

\section{CONCLUSIONS}

Psychiatric disorders from anxiety disorders to psychomotor agitation and/or delirium are often exacerbated by hospitalization and respiratory symptoms experienced by these patients as well as isolation. These factors make the management of these patients very challenging, both from pharmacological and clinical point of views. It should be considered that it is also very difficult to regularly supervise these patients because of their isolation with a consequent high risk of adverse events. Good sense should be used in order to package strategies aimed to buffer these factors and to reduce the risk of adverse events, never as much as in this context. In particular, in the absence of specific guidelines for the treatment of dementia and related symptoms, it is very important to have a personalized therapeutic approach that takes into account the symptoms, comorbidities, geriatric syndromes (such as, malnutrition, sarcopenia, delirium, pressure ulcers), drug interactions, compliance with treatment, and prognosis.

Potential strategies to better manage frail older subjects affected from mild to severe cognitive impairment can be:

- ability to make "smart cohorts": patients with dementia should be placed with young or not cognitive impaired, not very symptomatic patients in the room. "Proper roommates" act as "caregivers" useful in night and daytime surveillance, in helping eating meals or in repositioning respiratory devices properly. In addition, the presence of another person in the room makes the climate of the room familiar, softens the sense of loneliness which often lead these patients to get out of bed to look for someone, with high risk of falling and increases episodes of delirium as well as an increased number of accesses to the room by staff, resulting in overuse of personal protective equipment;

- during the visit or from outside the door frequent calls/videocalls with family members who could reassure the patient; this strategy seems also to be able to decrease the degree of space time disorientation;

- call always the patient by name, reminding him of some positive episodes from his past life. It will serve to be perceived as a "non-threatening presence".

In conclusion, as recommended by international dementia experts and Alzheimer's Disease International ${ }^{29}$, specific support for frail older people with any type of dementia and their families is immediately needed worldwide ${ }^{30,31}$.

\section{References}

1 Landi F, Barillaro C, Bellieni A, et al. The new challenge of geriatrics: saving frail older people from the SARS-CoV-2 pandemic infection. J Nutr Health Aging 2020. https://doi. org/10.1007/s12603-020-1356-X

2 Wang $H$, Barbarino P, Gauthier S, et al. Dementia care during COVID-19. Lancet 2020;Mar 30. pii: S01406736(20)30755-8

3 Jordan RE, Adab P, Cheng KK. COVID-19: risk factors for severe disease and death. BMJ 2020;368:m1198. 
4 Linnemann C, Lang UE. Pathways connecting late-life depression and dementia. Front Pharmacol 2020;11:279. https://doi.org/10.3389/fphar.2020.00279

5 Wiels W, Baeken C, Engelborghs S. Depressive symptoms in the elderly-an early symptom of dementia? A systematic review. Front Pharmacol 2020;11:34. https://doi. org/10.3389/fphar.2020.00034

6 Li Q, Guan X, Wu P, et al. Early transmission dynamics in Wuhan, China, of novel coronavirus-infected pneumonia. N Engl J Med 2020;382:1199-207.

7 Liu K, Chen Y, Lin R, et al. Clinical feature of COVID-19 in elderly patients: a comparison with young and middle-aged patients. J Infect 2020;Mar 11. pii: S0163-4453(20)30116X. https://doi.org/10.1016/j.jinf.2020.03.005

8 Chan JF, Yuan J, Kok KH, et al. A familial cluster of pneumonia associated with the 2019 novel coronavirus indicating person-to-person transmission: a study of a family cluster. Lancet 2020;395:514-23.

9 Bouadma L, Lescure FX, Lucet JC, et al. Severe SARSCoV-2 infections: practical considerations and management strategy for intensivists. Intensive Care Med 2020;46:57982. https://doi.org/10.1007/s00134-020-05967-x

10 Haffajee RL, Mello MM. Thinking globally, acting locally the U.S. response to COVID-19. N Engl J Med 2020;Apr 2. https://doi.org/10.1056/NEJMp2006740

11 de Camargo MC, Barros BCA, Fulone I, et al. Adverse events in patients with rheumatoid arthritis and psoriatic arthritis receiving long-term biological agents in a real-life setting. Front Pharmacol 2019;10:965. https://doi. org/10.3389/fphar.2019.00965

12 Mahase E. COVID-19: demand for dexamethasone surges as RECOVERY trial publishes preprint. BMJ 2020;Jun 23;369:m2512. https://doi.org/10.1136/bmj.m2512

13 Su B, Wang Y, Zhou R, et al. Efficacy and tolerability of lopinavir/ritonavir- and efavirenz-based initial antiretroviral therapy in HIV-1-infected patients in a tertiary care hospital in Beijing, China. Front Pharmacol 2019;10:1472. https://doi.org/10.3389/fphar.2019.01472

14 Bresciani E, Saletti C, Squillace N, et al. miRNA-218 targets Lipin-1 and glucose transporter type 4 genes in 3T3-L1 cells treated with lopinavir/ritonavir. Front Pharmacol 2019;Apr 30;10:461. https://doi.org/10.3389/ fphar.2019.00461

15 DiCenzo R, Peterson D, Cruttenden K, et al. Effects of valproic acid coadministration on plasma efavirenz and lopinavir concentrations in human immunodeficiency virus-infected adults. Antimicrob Agents Chemother 2004;48:4328-31.

16 Greenblatt DG, von Moltke LL, Harmatz JS, et al. Shortterm exposure to low-dose ritonavir impairs clearance and enhances adverse effects of trazodone. J Clin Pharmacol 2003;43:414-22.
17 Sampson MR, Cao KY, Gish PL, et al. Dosing recommendations for quetiapine when coadministered with hiv protease inhibitors. J Clin Pharmacol 2019;59:500-9.

18 Hsu AJ, Carson KA, Yung R, et al. Severe prolonged sedation associated with coadministration of protease inhibitors and intravenous midazolam during bronchoscopy. Pharmacotherapy 2012;32:538-45.

19 Yanofski J, Croarkin P. Choosing antidepressants for hiv and AIDS patients: insights on safety and side effects. Psychiatry 2008;5:61-6.

20 Timour Q, Frassati D, Descotes J, et al. Sudden death of cardiac origin and psychotropic drugs. Front Pharmacol 2012;3:76. https://doi.org/10.3389/fphar.2012.00076

21 Moss DM, Liptrott NJ, Siccardi M, et al. Interactions of antiretroviral drugs with the SLC22A1 (OCT1) drug transporter. Front Pharmacol 2015;6:78. https://doi. org/10.3389/fphar.2015.00078

22 Tsai FJ, Ho MW, Lai CH, et al. Evaluation of oral antiretroviral drugs in mice with metabolic and neurologic complications. Front Pharmacol 2018;9:1004.

23 Gao J, Tian Z, Yang X. Breakthrough: chloroquine phosphate has shown apparent efficacy in treatment of COVID-19 associated pneumonia in clinical studies. Biosci Trends 2020;14:72-3.

24 Yan Y, Zou Z, Sun Y, et al. Anti-malaria drug chloroquine is highly effective in treating avian influenza $\mathrm{A} \mathrm{H} 5 \mathrm{~N} 1$ virus infection in an animal model. Cell Res 2013;23:300-2.

25 Kassi EN, Papavassiliou KA, Papavassiliou AG. G6PD and chloroquine: selecting the treatment against SARS-CoV-2? J Cell Mol Med 2020;24:4913-4.

26 Boyadzhieva V, Stoilov N, Ivanova M, et al. Real world experience of disease activity in patients with rheumatoid arthritis and response to treatment with varios biologic DMARDs. Front Pharmacol 2018;9:1303. https://doi. org/10.3389/fphar.2018.01303

27 Landi F, Sieber C, Fielding RA, et al. Nutritional intervention in sarcopenia: report from the International Conference on Frailty and Sarcopenia Research Task Force. J Frailty Aging 2018;7:247-52.

28 Landi F, Calvani R. Protein and sarcopenia: experimental data and clinical evidence. Curr Protein Pept Sci 2018;19:632.

29 Alzheimer's Disease International. ADI offers advice and support during COVID-19 (https://www.alz.co.uk/news/ adi-offers-advice-and-support-during-covid-19).

30 Nilaweera D, Freak-Poli R, Ryan J. Impact of psychological stress and trauma on later-life cognitive function and dementia. Journal of Gerontology and Geriatrics 2019;67:114-22.

31 Giannotti C, Nencioni A, Odetti F, et al. Medication management ability in older patients: time for a reappraisal. Journal of Gerontology and Geriatrics 2019;67:8-12. 